\title{
Evolutionary denoising based on an estimation of Hölder exponents with oscillations.
}

\author{
Pierrick Legrand $^{1,2}$, Evelyne Lutton ${ }^{2}$ and Gustavo Olague ${ }^{1}$ \\ 1 CICESE, Research Center, Applied Physics Division \\ Centro de Investigación Científica y de \\ Educación Superior de Ensenada, B.C. \\ Km. 107 carretera Tijuana-Ensenada, \\ 22860, Ensenada, B.C. México \\ 2 INRIA Rocquencourt, Complex Team \\ Domaine de Voluceau BP 105 \\ 78153 Le Chesnay Cedex, France.
}

\begin{abstract}
In multifractal denoising techniques, the acuracy of the Hölder exponents estimations is crucial for the quality of the outputs. In continuity with the method described in [1], where a wavelet decomposition was used, we investigate the use of another Hölder exponent estimation technique, based on the analysis of the local "oscillations" of the signal. The associated inverse problem to be solved, i.e. finding the signal which is the closest to the initial noisy one but having the prescribed regularity, is then more complex. Moreover, the associated search space is of a different nature as in [1], which necessitates the design of ad-hoc genetic operators.
\end{abstract}

\section{Introduction}

In the past years many different signal and image denoising techniques have been proposed, some of them being even based on artificial evolution $[1,2]$. The basic notations are the following. One observes a signal or an image $Y$ which is some combination $F(X, B)$ of the signal of interest $X$ and a noise $B$. Making various assumptions on the noise, the structure of $X$ and the function $F$, one then tries to obtain an estimate $\hat{X}$ of the original signal, optimal in some sense. We consider denoising as equivalent to increasing the Hölder function $\alpha_{Y}$ (see section 2 for definitions) of the observations. Indeed, it is generally true that the local regularity of the noisy observations is smaller than the one of the original image, so that in any case, $\alpha_{X}$ should be greater than $\alpha_{Y}$.

In this paper, section 2 recalls some basic facts about Hölder regularity analysis. We describe in section 3 how oscillations are used to provide an estimator of the Hölderian regularity. The new denoising method is explained in section 4 and the evolutionary algorithm, with its ad-hoc genetic operators, are detailed in section 5. Numerical experiments are presented in section 6 . 


\section{Hölder regularity}

To simplify notations, we deal with $1 \mathrm{D}$ signals, and we assume that signals are nowhere differentiable. Generalisation to differentiable signals simply requires to introduce polynomials in the definitions [3]. Below the definitions of the pointwise and local Hölder exponents are given.

Let $\alpha \in(0,1)$, and $x_{0} \in K \subset R$. A function $f: K \rightarrow R$ is in $C_{x_{0}}^{\alpha}$ if for all $x$ in a neighbourhood of $x_{0},\left|f(x)-f\left(x_{0}\right)\right| \leq c\left|x-x_{0}\right|^{\alpha}(2)$ where $c$ is a constant. The pointwise Hölder exponent of $f$ at $x_{0}$, denoted $\alpha_{p}\left(f, x_{0}\right)$, is the supremum of the $\alpha$ for which (2) holds.

Let us now introduce the local Hölder exponent: Let $\alpha \in(0,1), \Gamma \in R$. One says that $f \in C_{l}^{\alpha}(\Gamma)$ if $\exists C: \forall x, y \in \Gamma: \frac{|f(x)-f(y)|}{|x-y|^{\alpha}} \leq C$ (3). Let $\alpha_{l}\left(f, x_{0}, \rho\right)=\sup \left\{\alpha: f \in C_{l}^{\alpha}(B(x 0, \rho))\right\}$. The local Hölder exponent of $f$ at $x_{0}$ is $\alpha_{l}\left(f, x_{0}\right)=\lim _{\rho \rightarrow 0} \alpha_{l}\left(f, x_{0}, \rho\right)$.

Since $\alpha_{p}$ and $\alpha_{l}$ are defined at each point, we may associate to $f$ two functions $x \rightarrow \alpha_{p}(f, x)$ and $x \rightarrow \alpha_{l}(f, x)$ which are two different ways of measuring the evolution of its regularity.

The quality of a denoising technique based on these exponents, strongly relies on the quality of an estimator of these quantities. In [1], the estimation was performed by a wavelet technique. We will see in the sequel that a better estimation of the Hölder exponent can be obtained by measuring the oscillations of the function.

\section{Estimation by oscillations}

The estimation based on oscillations measurements is a direct application of the local Hölder exponent definition (see [4]). The condition (3) can be written as: A function $f(t)$ is Hölderian with exponent $\alpha \in[0,1]$ at $t$ if there exists a constant $c$, for all $\tau: o s c_{\tau}(t) \leq c \tau^{\alpha}$ with $\operatorname{osc}_{\tau}(t)=\sup _{\left|t-t^{\prime}\right| \leq \tau} f\left(t^{\prime}\right)-\inf _{\left|t-t^{\prime}\right| \leq \tau} f\left(t^{\prime}\right)=\sup _{t^{\prime}, t^{\prime \prime} \in[t-\tau, t+\tau]}\left|f\left(t^{\prime}\right)-f\left(t^{\prime \prime}\right)\right|$. At each point we estimate the pointwise Hölder exponent as the slope of the regression of the logarithm of the oscillation versus the size of the window $\tau$. As we see in figure 1 , the estimation by oscillations provides better results than an estimation by wavelets.

\section{Method}

According to the notation of section 1 , we seek a denoised version $\hat{X}$ of the observed signal $Y$ that meets the following constraints:

1) $\hat{X}$ is close to $Y$ in the $L^{2}$ sense.

2) The Hölder function of $\hat{X}$ is prescribed.

If $\alpha_{X}$ is known, we choose $\alpha_{\hat{X}}=\alpha_{X}$. In some situations, $\alpha_{X}$ is not known but can be estimated from $Y$, see [5]. Otherwise, we just set $\alpha_{\hat{X}}=\alpha_{Y}+\delta$, where $\delta$ is a user-defined positive function, so that the regularity of $\hat{X}$ will be everywhere larger than the one of the observations. Two problems have to be solved in order to obtain $\hat{X}$. First, a procedure that computes the Hölder function of a signal from discrete observations is needed. 
Second, we need to be able to manipulate the data so as to get a specific regularity. To solve the first problem, the estimation method of section 3 is used, and for the second problem, an evolutionary algorithm has been designed.

\section{Evolutionary algorithm}

We consider that an individual is a signal (1D or $2 \mathrm{D})$. On the contrary to [1] where an individual was made of a subset of wavelet coefficients, a direct encoding of the signal in the genome has been used.

Initialisation: As the search space is extremely large, a direct search starting from a random set of initial signals has no chance to provide a good denoising in a reasonable time. However, many initial guess are available, including the noisy signal itself. We actually use several deterministic denoising methods to provide the initial population. These methods are the Multifractal Bayesian Denoising[6] and the Multifractal Pumping[5], and depend from a parameter setting. The parameters are generated randomly.

Fitness: An important point of the method is that the fitness calculation is based on two kind of fitness function. A pointwise fitness has been defined for each point of the signal as a combination between the quality of the individual in term of regularity and in term of distance to the noisy signal. As said above, it is based on the estimation by oscillations. The pointwise fitness is then combined to provide a local fitness. The local fitness is the sum of the pointwise fitness on a given segment (or window). The local fitness is used in the crossover and in the mutation operators. We compute the global fitness when we perform this sum on the full signal. This fitness is used for the selection and for the ranking. Crossover: A simple ranking selection mechanism with selective pressure 2 is used to select two individuals. Random crossing points are then selected. For images, a set of random rows and columns is chosen. The local fitness on each resulting segment is then used to select the best parts of the two individuals as the corresponding segment of the child.

Mutation: In a similar way, each segment (or image window) is muted using a probability law inversely proportional to the local fitness. For each individual we consider the worst local fitness wlf i.e. the fitness of the worst segment. Let $l f(j, i)$ the local fitness of the $i^{\text {th }}$ segment of the $j^{\text {th }}$ individual. The probability of mutation for this segment is $\operatorname{Pm}(j, i)=\frac{l f(j, i)}{w l f}$.

\section{$6 \quad$ Numerical Results}

For the first example (see figure 2), the original signal is a Generalized Weierstrass function with a regularity $\alpha(X, t)=t$ with $t \in[0,1]$. This signal is corrupted by a white Gaussian noise (standard deviation equal to 0.3 ). We use a synthetic image to perform an experiment in 2 dimensions. The figure 3 shows the original image, the noisy one, a denoising by Soft Thresholding and by our method. The second row displays the corresponding Hölder functions. As in the previous examples, our method 
allows to obtain a denoised version of the signal with the prescribed regularity.

\section{Conclusion}

We have experimented in this paper a new scheme for a multifractal denoising technique. It is based on a more precise and more complexcomputation of the Hölder exponent of a signal. This work is actually a first attempt to use an estimation of Hölder exponent based on oscillations for signal enhancement. Preliminary experiments yield satisfactory results, with a more precise control of the reconstructed regularity, which has to be considered as a major advantage for this type of techniques. Moreover, the evolutionary engine that has been designed has the following interesting characteristics: it performs a basic hybridisation with two other denoising techniques (Multifractal Bayesian Denoising and Multifractal Pumping for the initialisation step), and uses locally optimised genetic operators. Further work will first consist in a more precise analysis of the locally optimised genetic operators in comparison with classical "blind" ones. Second, the hybridisation scheme has to be investigated as it may be a good solution to reduce computation costs of the method. Additionally, the availability of a pointwise and local definition of the fitness opens the way to "Parisian" evolution implementations for the genetic engine. This may be another solution to reduce computational expenses of the method, see for example $[7,8]$.

\section{References}

1. J. Levy Vehel and E. Lutton, "Evolutionary signal enhancement based on hölder regularity analysis," EVOIASP2001, LNCS 2038, 2001.

2. Pierre Grenier, Jacques Levy Vehel, and Evelyne Lutton, "An interactive ea for mulifractal bayesian denoising," EvoIASP 2005, 30 March - 1 April, Lausanne, 2005.

3. Y. Meyer, "Wavelets, Vibrations and Scalings," American Mathematical Society, CRM Monograph Series, vol. 9, 1997.

4. C. Tricot, Curves and Fractal Dimension, Springer-Verlag, 1995.

5. P. Legrand, "Débruitage et interpolation par analyse de la régularité hölderienne. application à la modélisation du frottement pneumatique-chaussée, phd thesis," Université de Nantes et Ecole Centrale de Nantes, 2004.

6. J. Levy Vehel and P. Legrand, "Bayesian multifractal denoising," ICASSP 2003, IEEE internat. conf. on Acoustics, Speech, and Signal Processing, Hong Kong, April 6-10 2003.

7. Frederic Raynal, Pierre Collet, Evelyne Lutton, and Marc Schoenauer, "Polar ifs + parisian genetic programming = efficient ifs inverse problem solving," Genetic Programming and Evolvable Machines Journal, Volume 1, Issue 4, pp. 339-361, October, 2000.

8. Enrique Dunn, Gustavo Olague, and Evelyne Lutton, "Automated photogrammetric network design using the parisian approach," EvoIASP 2005, 30 March - 1 April, Lausanne, 2005. 


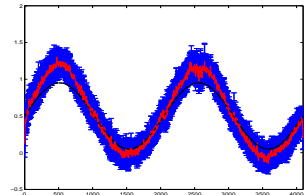

W1

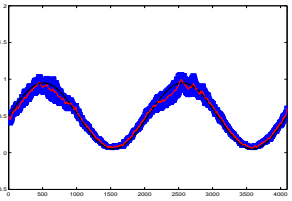

OSC

Fig. 1. 10 multifractional Brownian Motions have been built with a regularity $H$ evolving like a sine. The 2 methods of estimation of the Hölderian regularity have been applied: a wavelet-based (W1) and the method by oscillations (OSC). After an optimisation of the parameters of the 2 methods in term of risk, the means of the estimated Hölder functions are displayed.

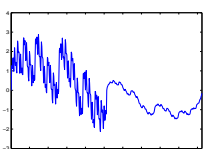

original

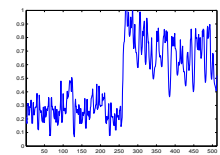

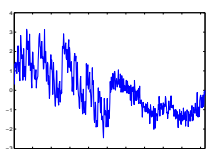

noisy

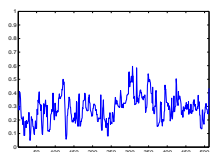

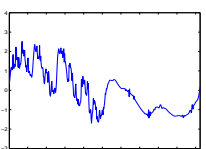

Soft Thres.

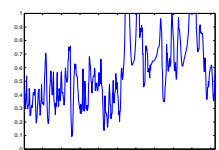

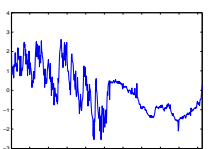

$10 \times 50$ ind

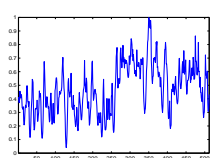

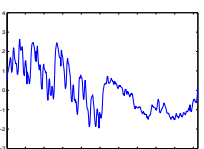

$500 \times 200$ ind

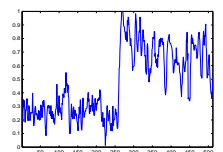

Fig. 2. First row: original Generalized Weierstrass Function, noisy version, denoising with Soft Thresholding, denoising by our method after 10 generations and 50 individuals, denoising by our method after 500 generations and 200 individuals. Second row: corresponding Hölder functions. Our method allow to recover almost perfectly the Hölder function of the original signal.
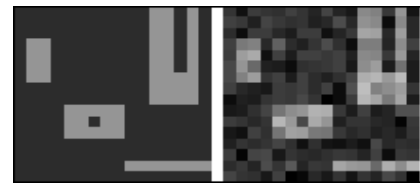

original

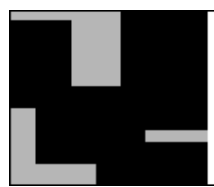

noisy

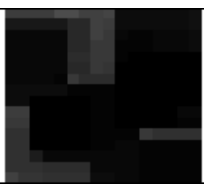

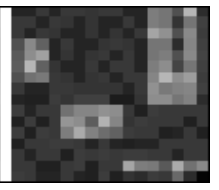

$500 \times 100$ ind
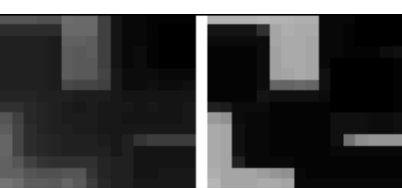

Fig. 3. Original image, the noisy one, a denoising by Soft Thresholding and by our method (100 ind, 500 gen). The second row displays the corresponding Hölder functions. 